\title{
Zum Forschungsstand über Ansätze des Dual Career Coachings
}

\author{
Philipp Ziegler-Rehak
}

Eingegangen: 26. Oktober 2020 / Angenommen: 25. November 2020 / Online publiziert: 13. April 2021 (C) Der/die Autor(en) 2021

Zusammenfassung Der vorliegende Artikel zeigt den Forschungsstand zur Beratung von Dual Career Couples (DCCs) anhand von zehn theoretisch, empirisch und praxeologisch entwickelten Ansätzen auf. Zunächst wird die Bedeutung des Phänomens DCCs für die Karriereberatung konturiert und anschließend vor dem Hintergrund gesellschaftlicher Transformationsprozesse eingeordnet. Die Einführung eines triadisch konzeptualisierten Beratungsraums schafft einen Rahmen, der eine vergleichende Darstellung und Einordnung der zehn Ansätze ermöglicht. Abschließend werden Perspektiven zur Weiterentwicklung des Dual Career Coachings aufgezeigt und eine Standortbestimmung vorgenommen.

Schlüsselwörter Dual Career Coaching · Dual Career Couples · Karriereberatung

Dipl.-Oec. P. Ziegler-Rehak

Institut für Psychologie, Universität Kassel, 34127 Kassel, Deutschland

Privatanschrift: Glücksburger Straße 8, 34225 Baunatal, Deutschland

E-Mail: kontakt@dualcareercoaching.de 


\title{
Approaches to Dual Career Coaching: Current state of research
}

\begin{abstract}
The article at hand summarizes the current state of research regarding the coaching of Dual Career Couples (DCCs) based on ten theoretically, empirically or praxeologically developed approaches. To get started the impact of the DCC phenomenon on career counseling is carved out, followed by a reflection with respect to societal transformational processes. The introduction of a triadically conceptualized counseling space creates a framework that subsequently allows a comparative presentation and classification of the ten approaches. Finally perspectives for the further development of Dual Career Coaching are illustrated to allow a localization of the current state of research.
\end{abstract}

Keywords Dual Career Coaching · Dual Career Couples · Career Counseling

\section{1 ,The most important career choice you'll make is who you marry“- Karriereberatung neu denken}

Seit sich Rapoport und Rapoport (1969) erstmals mit dem Phänomen der Dual Career Couples (DCCs) befasst haben, sind inzwischen 50 Jahre vergangen. Und trotzdem werden Jahr für Jahr neue Forschungsergebnisse publiziert, die sich sowohl als Beleg für den Facettenreichtum als auch die gesellschaftspolitische Relevanz dieses Themas ins Feld führen lassen. Doppelkarriere-Beziehungen sind im Wachstum und haben in vielen Ländern traditionelle Beziehungsmodelle als Norm abgelöst oder sind im Begriff, dies zu tun (Petriglieri 2019). Und trotz aller berechtigten Kritik daran, dass verschiedene Generationen oft allzu stereotypen Merkmalszuschreibungen anheimfallen, werden mit dem Übergang von vielen Millionen Baby-Boomern in den Ruhestand und der beruflichen Entwicklung der Millennials teils signifikante Veränderungen mit Blick auf arbeitsweltliche Werte, Motive und Kompetenzen sichtbar (Hardering 2018; Otto und Remdisch 2015; v. Weichs und Kauke 2019). Diese sind nicht zuletzt geprägt von einem wechselseitig veränderten Verständnis der Geschlechter hinsichtlich partnerschaftlicher Rollenverteilungen und daraus erwachsender beruflicher Entwicklungsmöglichkeiten und -restriktionen (Abouzahr et al. 2017). Karriereberatung muss vor dieser Hintergrundfolie aus meiner Sicht auf den Prüfstand gestellt und neu gedacht werden, um angemessen Bezug auf die arbeitsund lebensweltliche Wirklichkeit der Klientensysteme nehmen zu können.

Sheryl Sandberg, Chief Operating Officer von Facebook, sieht die Wahl eines Lebenspartners $^{1}$ als wichtigste Karriereentscheidung einer Person an: „The most important career choice you'll make is who you marry“ (Businessinsider 2011). Dass mit dem Zusammenspiel von Partnerschaft und Beruf verbundene Reflexionsund Aushandlungsprozesse in der arbeitsweltlichen Beratung an Bedeutung gewinnen werden, liegt für mich auf der Hand. Und ebenso, dass diese sich in einer Dual Career Konstellation wie unter einem Brennglas abbilden und erheblichen Einfluss

\footnotetext{
1 Um eine gendergerechte Schreibweise und den Lesefluss gleichermaßen sicherzustellen, werden im Folgenden wechselnd weibliche und männliche Schreibweisen verwendet.
} 
auf die berufliche Entwicklung einer Person nehmen können. Vor diesem Hintergrund ist es umso erstaunlicher, dass sich die Forschung zur Beratung von DCCs zurzeit noch auf eine überschaubare Anzahl von überwiegend theoretisch bzw. praxeologisch entwickelten Ansätzen beschränkt. Allein Petriglieri (2019) leitet ihre Befunde aus einer qualitativ-empirischen Untersuchung ab. Eine systematische Zusammenfassung des Forschungsstands, welche die vorliegenden Erkenntnisse ordnet und Perspektiven zur Entwicklung eines integrativen Dual Career Coaching-Ansatzes aufzeigt, wurde bislang nicht durchgeführt. Diese Lücke soll mit dem vorliegenden Artikel geschlossen werden.

\section{Dual Career - früher die Ausnahme, heute der Normalfall}

Rapoport und Rapoport (1969) initiierten die Forschung zum Phänomen der DCCs und definierten die Teilgruppe der Dual Career Familien als „FAMILIES IN which both husband and wife pursue careers (i.e. jobs which are highly salient personally, have a developmental sequence and require a high degree of commitment) and at the same time establish a family life with at least one child“ (S. 3). Im Kontrast zu den damaligen gesellschaftlichen Normen arbeiteten sie durch 16 Interviews mit DCCs progressive Rollenverteilungen und -bilder heraus, die auf eine stärkere Gleichberechtigung zwischen den Partnern abzielen und zugleich verschiedene Dilemmata verursachen können. Seitdem wurden viele Phänomenbereiche untersucht, unter anderem die berufliche Mobilität von DCCs, deren intrapartnerschaftliche Koordination oder DCC-spezifische Personalstrategien (Schulte 2002; Solga und Wimbauer 2005a).

Sowohl die Definitionen von DCCs als basalem Phänomen als auch die als relevant erachteten Phänomenbereiche sind stark durch die jeweiligen gesellschaftlichen, ökonomischen und kulturellen Rahmenbedingungen geprägt und durchlaufen im Wechselspiel mit diesen eine kontinuierliche Transformation. Ihre Veränderung von einer Partnerschaftsform mit Nischendasein zur neuen Normalität in der Arbeitswelt (Petriglieri und Obodaru 2019) hat zur Folge, dass DCCs inzwischen im Mainstream der strategischen HR-Themen angekommen sind und von großen Beratungsfirmen wie McKinsey, Boston Consulting Group oder Ernst \& Young mit eigenen breit angelegten Forschungsaktivitäten untersucht werden (Abouzahr et al. 2017; Azil et al. 2019; EY 2015). Deren Fokus liegt freilich vor allem auf der Entwicklung und Implementierung neuer personalpolitischer Strategien, um DCCs als Beschäftigte zu gewinnen und zu binden. Und weniger auf der Exploration neuer Methoden, die DCCs zur eigenverantwortlichen Arbeit an ihrem gemeinsamen Lebensentwurf befähigen. Insofern sind beratungswissenschaftliche Forschungsaktivitäten mit Blick auf Themen, die DCCs aktuell bewegen, eine wichtige Voraussetzung, um diesen Paaren eine Auseinandersetzung mit ihrem ,gesamten Lebensentwurf mit allen seinen Implikationen“ (Schreyögg 2013, S. 32) sowie dessen selbstbestimmte Gestaltung zu ermöglichen.

Es ist zu diskutieren, ob der Forschungsstand zur Beratung von DCCs die Transformation des Phänomens angemessen widerspiegelt. Insbesondere im englischen Sprachraum nehmen Ansätze zur Beratung von DCCs häufig unmittelbar Bezug 
auf die von Rapoport und Rapoport (1969) aufgezeigten Dilemmata (z.B. O'Neil et al. 1987; Stoltz-Loike 1992; Walker et al. 1987). Diese Prädetermination auf die initial herausgearbeiteten Phänomenbereiche führt nach meiner Einschätzung dazu, dass viele Ansätze aus dem englischen Sprachraum wenig neue Perspektiven in der Beratung von DCCs aufzeigen, sondern unterschiedliche Methoden auf bereits untersuchte Phänomenbereiche adaptieren. Inwiefern dadurch Themenbereiche fokussiert werden, die für DCCs heute in Beratungsprozessen relevant sind, bleibt mit Blick auf die zuvor skizzierten Transformationsprozesse unklar und zeigt bereits die grobe Richtung auf, in der Fortschritte bei der Entwicklung von spezifischen Beratungsansätzen für DCCs zu erwarten wären.

\section{Triadische Konzeptualisierung des Beratungsraums im Dual Career Coaching}

Im Folgenden zeige ich eine triadische Konzeptualisierung des Beratungsraums im Dual Career Coaching auf. Diese dient als Referenzpunkt für die vergleichende Einordnung der bislang entwickelten Ansätze in Abschn. 5 und 6.

Der Beratungsraum von DCCs lässt sich in zweifacher Hinsicht triadisch konzeptualisieren. Zum einen kann ,die Beratung selbst [...] als das Hinzukommen eines Dritten [...] verstanden werden“ (Busse und Tietel 2018, S. 9). Das DCC wird während des Beratungsprozesses Teil einer personalen Triade (Buchholz 1993; Goldbrunner 2004; Vogler 2011). Durch die Beratungsperson wird „die Spannung einer dritten Position“ (Busse und Tietel 2018, S. 64) aufgebaut und die Reflexivität des Klientensystems erhöht (Busse und Tietel 2018; Tietel 2006; Vogler 2011). Die Funktion des Coachs liegt darin, dem Paar alternative Wahrnehmungs-, Konstruktions- und Verhaltensmöglichkeiten aufzuzeigen und es auf diesem Weg über seine Selbstdiagnosen und eigenen Lösungsversuche hinauswachsen zu lassen. Zum anderen lässt sich der Beziehungstyp DCC auch inhaltlich als Triade konzeptualisieren. Schreyögg (2013) sieht die Herausforderung von DCCs im Kern darin, funktionale Beziehungsarrangements auszuhandeln um ,zwei Karrieren und ein Paar- bzw. ein Familienleben zu vereinbaren“ (Schreyögg 2013, S. 134). Denn erst wenn ,die Erfordernisse des Berufslebens beider Partner mit den Anforderungen des Familienoder Paarlebens in Übereinstimmung gebracht werden, entsteht eine Doppelkarriere. Diese Arbeit bezeichnen wir als Vereinbarkeitsmanagement" (Behnke und Meuser 2005, S. 130). Somit können die Paarbeziehung (inkl. eines möglichen Familienlebens mit Kindern), die Karriere von Partner A sowie die Karriere von Partnerin B als konstitutive inhaltliche Elemente des Systems DCC verstanden werden. Das Beziehungsarrangement verbindet die drei Elemente und kann als emergentes Produkt ihres Zusammenwirkens interpretiert werden (Schreyögg 2013; Solga und Wimbauer 2005b). Es umfasst praktische Arrangements zur Verteilung von Rollen, Ressourcen und Aufgaben sowie bewusste und unbewusste psychologische Arrangements, welche die Paardynamik beeinflussen (Behnke und Meuser 2005; Petriglieri 2019; Petriglieri und Obodaru 2019).

Abb. 1 zeigt meine Konzeptualisierung des Beratungsraums von DCCs. Das Format bezeichne ich im Folgenden als Dual Career Coaching. Die äußere Triade steht 


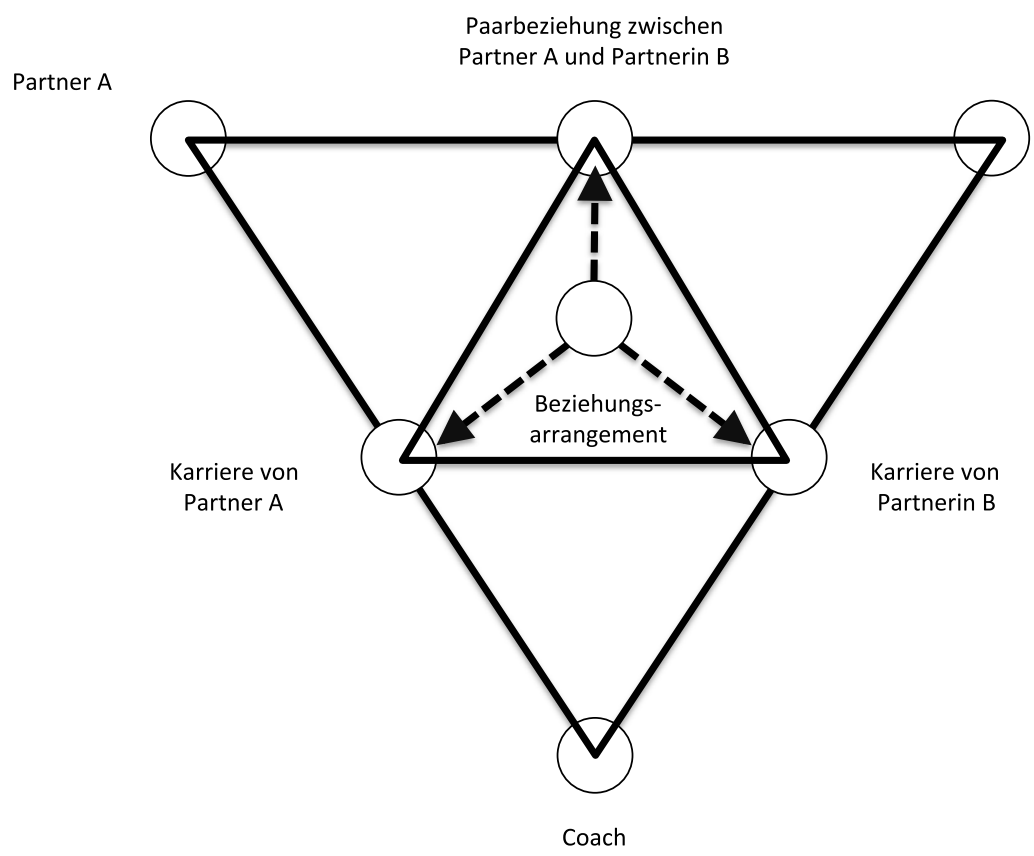

Partnerin B

Abb. 1 Beratungsraum im Dual Career Coaching. (Eigene Darstellung)

für die Erweiterung der dyadischen Beziehung des DCCs. Der Coach stärkt die Selbstreflexionsfähigkeit des Systems DCC, auf deren Basis das Beziehungsarrangement bewusst verändert oder bestätigt werden kann. Gegenstand der Reflexion ist die innere Triade.

\section{Methodisches Vorgehen zur Zusammenstellung des aktuellen Forschungsstands}

Die Recherche und Auswahl von Dual Career Coaching-Ansätzen orientierte sich am PRISMA-Schema für strukturierte Reviews (Moher et al. 2009). Im ersten Schritt wurde eine EBSCO-Suche mit Zugriff auf alle verfügbaren Datenbanken für die Suchbegriffe „Dual Career“ und „Coaching“ oder „Counseling“ bzw. „Counselling“ durchgeführt. Diese wurde durch eine Google Scholar-Suche über Publish or Perish ergänzt, um eine möglichst hohe Datensättigung zu erreichen. Außerdem wurde eine EBSCO-Suche für den Suchbegriff „,Doppelkarriere“ durchgeführt. Im zweiten Schritt wurden 172 EBSCO-Treffer sowie 680 Google Scholar-Treffer hinsichtlich ihrer Aussagekraft zum Dual Career Coaching gescreent (Beratungsrelevanz und Dual Career-Relevanz) und 100 Publikationen für das Elegibility Assessment ausgewählt. Im dritten Schritt wurde die spezifische Eignung dieser Publikationen als Dual Career Coaching-Ansatz simultan anhand folgender Kriterien überprüft: 
1. Es wurde überprüft, ob über die Benennung potenzieller Beratungsanliegen von DCCs und Implikationen für deren Beratung hinaus auch konkrete Aussagen zur Methodik im Beratungsprozess (z.B. Diagnose, Intervention) oder zum Beratungssetting (z.B. Rolle der Beratungsperson) getroffen und ob diese Aussagen von den Autorinnen selbst erarbeitet wurden (Weiterentwicklung des Forschungsstands). Neun der zehn final inkludierten Ansätze erfüllen dieses Selektionskriterium. Für den Ansatz von Petriglieri (2019) wurde auf dessen Anwendung verzichtet, da die Ergebnisse dieser empirischen Studie meines Erachtens auch ohne expliziten beratungswissenschaftlichen Bezug auf den Beratungskontext adaptiert werden können. Dieses Kriterium stellt sicher, dass die final inkludierten Publikationen konkrete Ansätze im Sinn einer methodisch fundierten Arbeit am Anliegen des Klientensystems aufzeigen.

2. Es wurde überprüft, ob Coaching oder Therapie fokussiert wird. Da Coaching laut Greif (2008) ,nicht zur psychotherapeutischen Beratung oder gar Therapie psychischer Störungen“ (S. 63) dient, ist meines Erachtens auch hier eine klare Grenzziehung indiziert. Diese wurde vorliegend durch die Exklusion aller Publikationen mit therapeutischem Fokus vorgenommen.

3. Es wurde überprüft, ob die Publikation die gemeinsame Beratung beider Partner konzeptionell erfasst bzw. zulässt. Alle final inkludierten Ansätze erfüllen dieses Selektionskriterium. Dieses Kriterium stellt sicher, dass die Ansätze mit der äußeren Triade des Beratungsraums im Dual Career Coaching kompatibel sind.

4. Es wurde überprüft, ob die Publikation über spezifische Phänomene einzelner Kulturräume hinaus aussagekräftig ist. Neun der zehn Ansätze erfüllen dieses Selektionskriterium uneingeschränkt. Der Ansatz von Hall (2018) besteht zwar überwiegend aus einer Case Study mit Spezifika eines bestimmten Kulturraums, weist durch die Übertragung des Life Design Paradigmas auf die Beratung von DCCs jedoch gleichzeitig eine interkulturelle Perspektive auf und wurde daher inkludiert. Dieses Kriterium stellt eine angemessene Reichweite der Ansätze durch Anschlussfähigkeit an möglichst viele Kulturräume sicher.

Nach Anwendung der Kriterien wurden sieben Artikel aus wissenschaftlichen Fachjournals, zwei bei wissenschaftlichen Fachverlagen erschienene Monografien (Schreyögg 2013; Stoltz-Loike 1992) und eine auf einer empirischen Forschungsarbeit basierende Monografie (Petriglieri 2019) selektiert. Im vierten Schritt wurde auf Basis des in Abschn. 3 beschriebenen Beratungsraums eine qualitative Synthese dieser zehn Ansätze durchgeführt. Diese werden nachfolgend chronologisch vorgestellt. Nach Zusammenfassung der Kernaussagen des jeweiligen Ansatzes zeige ich auf, welche Elemente des Beratungsraums im Dual Career Coaching durch den jeweiligen Ansatz abgedeckt werden. 


\section{Ansätze des Dual Career Coachings}

\subsection{O’Neil et al. (1987): Durch Karrieretransitionen ausgelöste Dynamiken erkennen und bewältigen}

O’Neil et al. (1987) entwickeln ein Assessment-Modell für die Eingangsdiagnose in der Beratung von DCCs, das in Abb. 2 dargestellt ist. Zentrales Konstrukt sind die Karrieretransitionen der Partnerinnen. Aufbauend auf dem Werk von Super (1957) benennen die Autoren 23 potenzielle Karrieretransitionen, deren Durchlaufen interpersonelle Dynamiken im System DCC auslöst. Im Assessment wird exploriert, welche Karrieretransitionen bereits durchlaufen wurden, aktuell anstehen oder für die Zukunft absehbar sind. Daran anknüpfend wird reflektiert, welche individuellen Entwicklungsbereiche für jede Partnerin von Bedeutung sind und welche Anpassungsleistungen, Coping-Strategien oder sonstigen Veränderungsbedarfe fokussiert werden müssen, damit das Gesamtsystem aus zwei Karrieren, Genderrollen und Paarbeziehung bzw. Familie in Balance bleiben kann. Das Zusammenspiel von Karrieretransitionen und individuellen Entwicklungsbereichen kann dazu führen, dass die von Rapoport und Rapoport (1969) herausgearbeiteten normativen Dilemmata in den Bereichen Karriere, Genderrollen und Paarbeziehung/Familie salient werden. Aus der individuellen Kombination von Karrieretransitionen, Entwicklungsbereichen und normativen Dilemmata ergeben sich spezifische normative Themen des DCCs, die sich in psychologischen und persönlichen Fragestellungen der Partnerinnen äußern, welche im Beratungsprozess zu bearbeiten sind.

Mit Blick auf die innere Themen-Triade im Dual Career Coaching fokussiert der Ansatz ausgehend von der dyadischen Beziehung zwischen den beiden Karrieren auch deren dyadischen Beziehungen zur Paarbeziehung des DCCs.

\subsection{Walker et al. (1987): Niedrige Paar-Umwelt-Passung erkennen und bewältigen}

Walker et al. (1987) greifen ebenfalls die von Rapoport und Rapoport (1969) herausgearbeiteten Dilemmata auf. Für die Eingangsdiagnostik schlagen sie eine Werteanalyse vor, um zu explorieren, ob und in welchem Ausmaß innerpartnerschaftliche Spannungen, Konflikte oder andere arbeits- und lebensweltliche Probleme des DCCs auf Diskrepanzen zwischen seinem Lebensmodell und institutionellen Policies zurückzuführen sind. Da sich sowohl individuelles Verhalten als auch gesellschaftliche und organisationale Policies auf Werte beziehen, können zu starke Diskrepanzen normative Dilemmata auslösen, die als Stressoren wirken. Hat das DCC ein dysfunktionales Ursache-Wirkungs-Verständnis, kann es zur Internalisierung des psychischen Drucks kommen, der sich in Konflikten zwischen den Partnern und gegenseitigem Victim Blaiming entlädt. Durch die Werteanalyse soll das DCC ein besseres Verständnis davon entwickeln, wie Institutionen die Umsetzbarkeit seines Beziehungsmodells und damit seine Lebensrealität beeinflussen. Sofern eine zu geringe Paar-Umwelt-Passung Ursache des Beratungsanliegens ist, können im weiteren Beratungsprozess angemessene Paar-Umwelt-Coping-Strategien erarbeitet 





oder Aktivitäten zur Beeinflussung der institutionellen Policies entwickelt werden, um die Paar-Umwelt-Passung zu verbessern.

Mit Blick auf die innere Themen-Triade im Dual Career Coaching fokussiert der Ansatz eine Ebene, die ich im Beratungsraum bislang nicht eingeführt habe: die Umwelt des DCCs und die Wechselbeziehungen zwischen diesen beiden Systemen.

\subsection{Stoltz-Loike (1992): Kompetenz zu gemeinsamer Beziehungs- und Karrieregestaltung entwickeln}

Stoltz-Loike (1992) stellt nach meiner Einschätzung den bislang umfassendsten Ansatz zur Beratung von DCCs vor. Dieser soll DCCs befähigen, eine Balance zwischen den Verantwortlichkeiten und Rollen der Partner herzustellen. Die Interventionsstrategie ist auf die Entwicklung von Meta-Kompetenzen wie Kommunikations-, Verhandlungs- und Konfliktlösungsfähigkeit ausgerichtet. Der Ansatz soll den Coach befähigen, angemessene Diagnosen zu stellen und zielführende Entwicklungsprozesse beim DCC anzustoßen. Im Beratungsprozess soll eine Stärkung der Kontrollüberzeugung beider Partnerinnen mit Blick auf die eigenen Lebensumstände erreicht werden, indem gemeinsame Ziele und ausgewogene Rollenverteilungen ausgehandelt oder angepasst werden. Eine dauerhaft als ungleich empfundene Verteilung wird als Ursache negativer psychischer Zustände und Affekte angesehen. Der Ansatz deckt die Einschätzung der Familien- und Karriereprioritäten, die Entwicklung von Kommunikations- und Verhandlungskompetenzen, den Umgang mit Konflikten und die Lebensspannen-Sichtweise auf Erfolg ab.

Mit Blick auf die innere Themen-Triade im Dual Career Coaching nimmt der Ansatz zwar auf alle drei Elemente Bezug, fokussiert dabei jedoch immer wieder das Beziehungsarrangement, das durch die Beratung in Balance gebracht bzw. gehalten werden soll.

\subsection{Spiker-Miller und Kees (1995): Karriere- und Paar-/Familienberatung zu einem integrierten Ansatz kombinieren}

Spiker-Miller und Kees (1995) arbeiten drei Bereiche heraus, in denen Probleme entstehen und mit dem Beziehungsmodell des DCCs interagieren können: (1) Konflikte bei der Priorisierung der beiden Karrieren im Verhältnis zueinander, insbesondere wenn die Karrierewünsche des einen Partners die Karrieremöglichkeiten der anderen Partnerin einschränken; (2) die Rollen- und Machtverteilung sowie den Grad der Zufriedenheit mit der Paarbeziehung und dem Familienleben im Familiensystem; und (3) individuelle Genderrollenkonflikte der einzelnen Partner. Des Weiteren wird aufgezeigt, welchen Einfluss die eigenen Werte und Einstellungen des Coachs zu unterschiedlichen Lebensstilen und -entwürfen auf den Beratungsprozess haben können. Der Kern des Beitrags besteht darin, die Notwendigkeit der Entwicklung eines integrierten Beratungsansatzes für DCCs zu begründen, der Karriereberatung mit Paar- und Familienberatung kombiniert und die für die jeweiligen Formate spezifischen Dynamiken berücksichtigt.

Mit Blick auf die innere Themen-Triade im Dual Career Coaching bleibt der Ansatz relativ unbestimmt, da er verschiedene Handlungsfelder und daraus resultie- 
rende Implikationen für die Gestaltung von Beratungsprozessen für DCCs aufzeigt, ohne daraus jedoch konkrete Vorschläge zur Gestaltung von Diagnostik und Interventionen abzuleiten. Mit Blick auf die äußere Triade markiert er den Einfluss normativer Haltungen des Coaches auf den Beratungsprozess.

\subsection{Clement und Clement (2001): Paardynamiken als Auslöser dysfunktionaler Beziehungs- und Karrieregestaltung aufdecken und bearbeiten}

Clement und Clement (2001) kontrastieren DCC-Beziehungen gegen die traditionelle Ehe. Beide Modelle haben spezifische Strukturen, Kosten und Gewinne. In der traditionellen Ehe mit ihrer komplementären Struktur kann das Paar auf kulturell vordefinierte Rollen und Aufgabenverteilungen zurückgreifen. Dadurch sind weniger Aushandlungsprozesse nötig. Das Modell erzeugt starke wechselseitige Abhängigkeiten und weist Wertigkeitsunterschiede zwischen den Rollen auf. DoppelkarriereBeziehungen haben eine symmetrische Struktur und ermöglichen den Partnerinnen mehr Autonomie und eine größere gegenseitige Feldkompetenz. Der Mangel an kulturell vordefinierten Lösungsmustern und die modellinhärente Zeitknappheit machen regelmäßige Aushandlungsprozesse nötig. Daraus resultieren Konfliktpotentiale auf den Achsen Innen/Außen und Innen/Innen.

Die erste Achse ist für eine Zeitkonkurrenz zwischen Familien- und Arbeitssphäre anfällig, da beide Partner Zeit in berufliche Rollen investieren müssen, die für die Familie nicht mehr zur Verfügung steht. Ein Kulturkonflikt, der darin besteht, dass die Mitgliedschaftsbedingungen von Organisationen stets aufs Neue zu erfüllen sind, während die Familienmitgliedschaft grundsätzlich bedingungslos ist, kann die Zeitkonkurrenz verschärfen und den Zugriff auf Familienzeit für berufliche Zwecke provozieren. Bei geringer bzw. abnehmender emotionaler Bindung des Paares kann die Nichterfüllung privater Verpflichtungen schnell zur Bilanzierung von „Geben und Nehmen“ (Clement und Clement 2001, S. 267) führen. Diesbezüglich empfundene Dysbalancen führen zu weiteren Konflikten und erschweren eine gemeinsamkeitsorientierte Beziehung mit der Möglichkeit zu geben, ohne unmittelbar nehmen zu wollen und langfristige Win-Win-Ergebnisse zu erzielen. Werden individuelle Bedürfnisse im Arrangement nicht ausreichend berücksichtigt, können Krisen und Konflikte schnell von der Aufkündigung des Arrangements bis hin zur Auflösung der Beziehung führen.

Die zweite Achse bildet paardynamische Phänomene ab und ist für Konfliktverschiebungen anfällig. In DCCs haben beide Partnerinnen häufig eine Vorliebe für Gestaltungsaufgaben, deren Erledigung auffällt, gegenüber ,Status-Quo sichernden Aufgaben“ (Clement und Clement 2001, S. 259), deren Nicht-Erledigung auffällt. Eine komplett symmetrische Aufgabenverteilung ist dauerhaft meist nur schwer aufrechtzuerhalten und kann bei Dysbalancen Konfliktdynamiken auslösen, durch die primäre Konfliktursachen der Innen/Außen-Achse als sekundäre Konflikte auf der Innen/Innen-Achse ausgetragen werden. Weitere Konfliktdynamiken können durch die Regression in tradierte Rollenmuster oder das Treffen von Karriereentscheidungen mit primärem Fokus auf die Paardynamik entstehen. 
Mit Blick auf die innere Themen-Triade im Dual Career Coaching fokussiert der Ansatz ausgehend vom Element der Paarbeziehung und dessen dyadischen Beziehungen zu den beiden Karrieren die Balance des Beziehungsarrangements.

\subsection{Parker und Arthur (2004): Intersubjektive Karrierekonstruktion bewusst machen und Karrieren bewusst gestalten}

Parker und Arthur (2004) stellen einen Ansatz zur Erfassung der intersubjektiv durch das DCC konstruierten Doppelkarriere vor. Anhand der inneren Karriere sowie des „intelligent career framework“ (Parker und Arthur 2004, S. 7) mit den Dimensionen knowing why, knowing what und knowing who entwickeln sie ein Card-Sorting-Verfahren für die Eingangsdiagnostik. Beide Partner wählen Karten aus, deren Aussagen ihnen in Bezug auf ihre eigene Lebenssituation bedeutsam erscheinen, und priorisieren diese. Aus den Ergebnissen werden zwei individuelle Reports und ein PaarReport generiert, um die beiden intrasubjektiven Karrierekonstruktionen der Partnerinnen sowie die intersubjektive Karrierekonstruktion des DCCs offenzulegen. Dann reflektiert das DCC, welche Faktoren seine Karrieregestaltung bislang bewusst oder unbewusst beeinflusst haben. Der Coach reduziert seine Rolle hierbei auf das Halten des Dialograumes. Anhand der herausgearbeiteten Kernthemen entwickelt das DCC gemeinsame Veränderungsanliegen und hält diese in einem Aktionsplan fest, der entlang der drei Karrieredimensionen des Intelligent Career Framework strukturiert ist.

Mit Blick auf die innere Themen-Triade im Dual Career Coaching fokussiert der Ansatz primär die dyadische Beziehung zwischen den beiden Karrieren und sekundär die dyadischen Beziehungen zwischen den beiden Karrieren und der Paarbeziehung.

\subsection{Jahn (2012): Rolle als organisationsinterner Dual Career Coach ausbalancieren}

Jahn (2012) konturiert im Kern die Rolle des internen Coachs, der im Auftrag der Organisation Beschäftigte in Doppelkarrierebeziehungen berät. Ausgehend von den intimen Einblicken des Coachs in die familiäre Situation des DCCs und seiner gleichzeitigen beruflichen Beziehung zu mindestens einer Partnerin wird das spezifische Spannungsfeld dieser Beratungskonstellation aufgezeigt. Dieses macht eine klare Verständigung über Grenzen in Bezug auf die zu beratenden Themen zu Beginn des Beratungsprozesses notwendig.

Mit Blick auf die äußere personale Triade im Dual Career Coaching zeigt der Ansatz spezifische Risiken auf, die durch Rolleninterferenzen des Coachs bei organisationsinternen Dual Career Coachings zum Tragen kommen können.

\subsection{Schreyögg (2008 und 2013): Beziehung und Karrieren mit Blick auf den angestrebten Lebensentwurf gestalten}

Schreyögg (2008) greift den Ansatz von Clement und Clement (2001) auf und entwickelt ihn in ihrem Buch ,Familie trotz Doppelkarriere. Vom Dual Career zum Dual Care Couple“ (2013) zu einem Life-Coaching-Format für Doppelkarriere-Fa- 
milien weiter. In der Analyse der Liebesbeziehung, in der Arbeit an einer guten „Balance von Geben und Nehmen“ sowie in einer guten „Organisation des Privaten“ und einem verlässlichen „Umgang mit Zeit“ sieht Schreyögg (2008, S. 399f.) die zentralen Fragestellungen in der Beratung von DCCs. Diese sind aus ihrer Sicht in der Beratung mit Blick auf „den gesamten Lebenszusammenhang“ (ebd., S. 397) und das Führen eines glücklichen Lebens durchzuarbeiten. Ihr Format ist auf den bewussten Umgang mit dem Engpassfaktor Zeit ausgerichtet, der vor allem für Doppelkarriere-Familien mit kleinen Kindern relevant ist. Die Autorin schlägt eine lebensbiografische Rahmung zu Beginn des Beratungsprozesses vor, um Rollenvorbilder sowie normative Werthaltungen und Rollenvorstellungen des DCCs offen zu legen und in Beziehung zum angestrebten Lebensentwurf zu setzen. Der Kern des Formats liegt laut Schreyögg (2013, S. 205) darin, die Lebensbereiche „Beruf, Familie, Partnerschaft, Bedürfnisse der einzelnen Familienmitglieder" durch die bewusste Gestaltung und gezielte Aushandlung phasenadäquater Arrangements (u.a. zu Aufgabenverteilung, Geld, Mobilität, Kinderbetreuung) ,in eine gute Balance“ zu bringen und zu halten.

Mit Blick auf die innere Themen-Triade im Dual Career Coaching fokussiert der Ansatz vor allem die Gestaltung des Beziehungsarrangements und damit die triadische Beziehung der drei Elemente.

\subsection{Hall (2018): Gemeinsames Life-Design durch Narrative konstruieren}

Hall (2018) überträgt den Life-Design-Ansatz von Savickas (2015) auf die Beratung von DCCs. Das für Individuen entwickelte Career Construction Interview (CCI) zur Exploration von Vorbildern, Lieblingsmedien, Lieblingsgeschichten, Lieblingssprichwörtern und favorisierten frühen Erinnerungen wird auf die Arbeit mit Paaren adaptiert. Durch den Einsatz des CCI sollen thematische Zugänge zur Lösung von Dilemmata, die sich dem DCC mit Blick auf bevorstehende Lebens-Karriere-Transitionen stellen, eröffnet werden. Der Beratungsprozess ist in vier Phasen gegliedert: (1) In der Konstruktionsphase werden mit den Antworten im CCI Mikronarrative evoziert, um die vom DCC konstruierten Lebens-Karriere-Umstände und damit verbundene Dilemmata zu veranschaulichen. (2) In der Dekonstruktionsphase deckt das DCC durch reflektive Beobachtung und Selbst-Exploration mit Blick auf die Mikronarrative Muster in seinen Lebensthemen sowie Annahmen und Glaubenssätze auf. (3) In der Rekonstruktionsphase werden die zuvor gewonnenen Erkenntnisse mit Blick auf die Identität und die aktuelle Situation des DCCs in neue Gestaltungsziele für das gemeinsame Leben und die beiden Karrieren überführt. (4) In der CoKonstruktionsphase erarbeitet der Coach mit dem DCC einen Aktionsplan, durch den die zuvor formulierten Gestaltungsziele in die Tat umgesetzt werden sollen.

Mit Blick auf die innere Themen-Triade im Dual Career Coaching fokussiert der Ansatz die aktive Gestaltung und Transformation der Paarbeziehung, der beiden Einzelkarrieren sowie ihrer wechselseitigen Bezüge. 


\subsection{Petriglieri (2019): Paar-Transitionen durch psychologische Arrangements gemeinsam bewältigen}

Der Ansatz von Petriglieri (2019) soll DCCs befähigen, ihre Beziehungen und professionellen Identitäten eigenverantwortlich zu gestalten. Identitäts- und Selbstkonstruktion basiert auf den lebens- und arbeitsweltlichen Erfahrungen einer Person und ist in DCC-Beziehungen ein hoch interdependenter Prozess. Die Autorin arbeitet mit Blick auf die professionelle Identitätskonstruktion von DCCs aus 113 qualitativen Paar-Interviews drei zentrale Paar-Transitionen als paarübergreifendes Muster heraus.

Die erste Transition fasst Petriglieri (2019, S. 31) mit „How Can We Make This Work?" zusammen. Ereignisse, die aus zwei unabhängigen Karrieren zwei interdependente Karrieren machen, führen zu einem erhöhten Koordinationsbedarf zwischen den Partnern. DCCs handeln oft einen Couple Contract, also ein Beziehungsarrangement, aus. Aus Sicht der Autorin sollte das DCC nicht nur praktische Arrangements zur Rollen-, Aufgaben- und Ressourcenverteilung vereinbaren, sondern auch über Werte, Ängste und Grenzen der Partner sprechen. Sie bestimmen oft die Entwicklungsrichtung und erleichtern als Kriterien das Treffen schwieriger Entscheidungen, z.B. indem sich das DCC bewusst auf ein Modell der Karrierepriorisierung verständigt.

Die zweite Transition fasst Petriglieri (2019, S. 110) mit „What Do We Really Want?“" zusammen. Sie tritt häufig während der Midlife Crisis bzw. der Individuation auf, wenn sich der Fokus der Partner in der Gestaltung ihres Lebens, ihrer Beziehung und ihrer Karriere vom Erfüllen fremder Erwartungen hin zum Realisieren eigener Wünsche verschiebt. Durch die hohe Karrieresalienz von DCCs ist damit häufig eine professionelle Neuerfindung einer Partnerin verbunden. Eine bidirektional sichere Bindung des Paares fördert in dieser Phase die Balance zwischen Geben und Nehmen von Unterstützung. Die Transition kann eine Neuaushandlung des Couple Contracts und dabei neben inhaltlichen Konflikten auch emotionale Paardynamiken auslösen.

Die dritte Transition fasst Petriglieri (2019, S. 176) mit „Who Are We Now?“ zusammen. Rollenwechsel und der nahende Ausstieg aus dem Berufsleben stellen DCCs vor die Herausforderung, ihre individuellen und kollektiven Identitäten zu erneuern. Zunächst müssen die Partner ihren Frieden mit ungelösten Entwicklungsaufgaben aus den ersten Transitionen machen, um dann eine Idee darüber entwickeln zu können, wie sie als Paar ihr Leben und ihre Beziehung ohne die Karrieren, die bislang in ihrem Lebensentwurf eine zentrale Rolle eingenommen haben, gemeinsam gestalten wollen.

Mit Blick auf die innere Themen-Triade im Dual Career Coaching fokussiert der Ansatz am deutlichsten die dyadische Beziehung zwischen den beiden Karrieren sowie die Gestaltung des Beziehungsarrangements und damit die triadische Beziehung der drei Elemente. 


\section{Perspektiven zur Weiterentwicklung des Dual Career Coachings}

Wie lassen sich nun die vorgestellten Ansätze vor der Hintergrundfolie des triadisch konzeptualisierten Beratungsraums im Dual Career Coaching einordnen?

Zunächst gibt es aus meiner Sicht zurzeit keinen Ansatz, mit dem sich alle Elemente des Beratungsraums adressieren lassen. Somit besteht die nächstliegende Perspektive darin, einen Ansatz zu entwickeln, der in Beratungsprozessen von DCCs grundsätzlich Themen aus dem gesamten Beratungsraum besprechbar macht und die Wahl von Setting, Methoden und zeitlicher Dauer der Beratung am konkreten Beratungsanliegen des DCCs ausrichtet (Schreyögg 2012). Spiker-Miller und Kees (1995) weisen mit ihrer Forderung nach einem integrierten Beratungsansatz in eine ähnliche Richtung, bieten jedoch keine praktische Lösung an.

Eine weitere Perspektive sehe ich darin, durch die Entwicklung einer Eingangsdiagnostik für das Dual Career Coaching künftig eine strukturierte Exploration des Klientensystems zu ermöglichen und sich dadurch systematisch dem Beratungsanliegen und den Interventionsschwerpunkten in der inneren Themen-Triade zu nähern (Möller und Kotte 2013). In Verbindung damit kann auch die Indikation fakultativer Diagnoseinstrumente, welche die oben genannten Ansätze für bestimmte Elemente im Beratungsraum bereitstellen, geprüft werden.

Mit Blick auf verbindende Elemente zwischen den einzelnen Ansätzen fällt auf, dass in den Monografien vor allem das Beziehungsarrangement eine zentrale Rolle spielt (Petriglieri 2019; Schreyögg 2013; Stoltz-Loike 1992). Die in Fachartikeln dargestellten Ansätze fokussieren oft hingegen stärker einzelne Elemente der inneren Themen-Triade bzw. deren Beziehungen zueinander, teils unter Berücksichtigung von Rückwirkungen auf das Beziehungsarrangement (Clement und Clement 2001; Hall 2018; O’Neil et al. 1987; Parker und Arthur 2004). In mehreren Fällen werden sowohl in Fachartikeln als auch in Monografien die Konstrukte der Transition (Hall 2018; O’Neil et al. 1987; Petriglieri 2019) und des Selbstkonzepts bzw. der Identität (Hall 2018; Parker und Arthur 2004; Petriglieri 2019) zur Konzeptualisierung der untersuchten Modelle genutzt.

Wie in Abb. 3 dargestellt, lassen sich diese Konstrukte als verbindende Elemente in den Beratungsraum im Dual Career Coaching integrieren und geben diesem eine dynamische Perspektive, da ihre Veränderung einerseits als Ausgangspunkt für Dysbalancen und andererseits als Ansatzpunkt zur Wiederherstellung bzw. Aufrechterhaltung des Gleichgewichts im System DCC gesehen werden kann. Somit besteht meines Erachtens eine forschungsbezogene Perspektive darin, künftige Konzeptualisierungen ebenfalls auf Basis dieser Elemente und Konstrukte vorzunehmen, um die Rückkopplung der Forschungsergebnisse an vorhandene Ansätze sowie ihre Integration in einen übergreifenden Ansatz zu erleichtern. Die Annahmen über die Zusammenhänge zwischen den Konstrukten, welche den theoretisch bzw. praxeologisch entwickelten Ansätzen zugrunde liegen, haben nach derzeitigem Stand den Charakter vorläufiger Befunde, deren empirische Überprüfung aussteht.

Während die innere Triade des Beratungsraums im Dual Career Coaching mit unterschiedlichen Schwerpunktsetzungen durch alle Ansätze fokussiert wird, spielt die äußere Triade aus Coach und den beiden Partnerinnen nur in zwei Ansätzen eine nennenswerte Rolle (Jahn 2012; Spiker-Miller und Kees 1995). Insofern bietet 


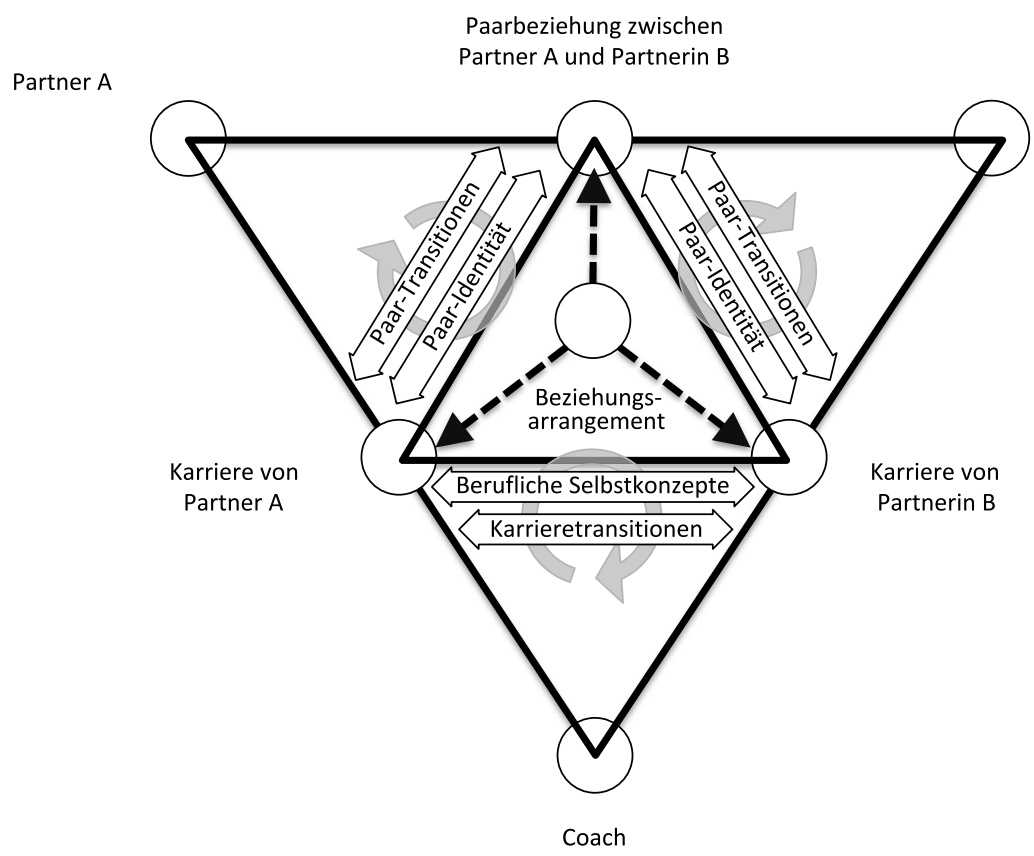

Partnerin B

Abb. 3 Dynamischer Beratungsraum im Dual Career Coaching. (Eigene Darstellung)

schließlich auch die tiefergehende Untersuchung der personalen Triade für mich eine vielversprechende Perspektive, um die spezifische Dynamik in der Beratung von DCCs noch besser zu verstehen und Beratungsprozesse wirksam zu gestalten.

\section{Fazit: Karriereberatung neu denken}

Doppelkarrierepartnerschaften haben sich in vielen Gesellschaften zum Normalfall entwickelt oder sind im Begriff, dies zu tun. Somit wäre ein integrativer Dual Career Coaching-Ansatz ein zentraler Beitrag, um Karriereberatung im Sinne meines eingangs formulierten Plädoyers neu zu denken. Die systematische Zusammenfassung des Forschungsstands, die ich mit dem vorliegenden Artikel angestrebt habe, zeigt: Es existiert ein solides Fundament von Ansätzen, die bereits spezifische Aspekte des Beratungsraums im Dual Career Coaching aufgreifen und in einen integrativen Ansatz einbezogen werden können, wenn auch größtenteils unter dem Vorbehalt, dass eine empirische Überprüfung der zugrunde liegenden Annahmen aussteht. Gleichzeitig kann bislang keiner der Ansätze den Anspruch eines integrativen Ansatzes erheben. Einen solchen zu entwickeln, scheint mir ein lohnendes beratungswissenschaftliches Unterfangen.

Funding Open Access funding enabled and organized by Projekt DEAL.

Open Access Dieser Artikel wird unter der Creative Commons Namensnennung 4.0 International Lizenz veröffentlicht, welche die Nutzung, Vervielfältigung, Bearbeitung, Verbreitung und Wiedergabe in 
jeglichem Medium und Format erlaubt, sofern Sie den/die ursprünglichen Autor(en) und die Quelle ordnungsgemäß nennen, einen Link zur Creative Commons Lizenz beifügen und angeben, ob Änderungen vorgenommen wurden.

Die in diesem Artikel enthaltenen Bilder und sonstiges Drittmaterial unterliegen ebenfalls der genannten Creative Commons Lizenz, sofern sich aus der Abbildungslegende nichts anderes ergibt. Sofern das betreffende Material nicht unter der genannten Creative Commons Lizenz steht und die betreffende Handlung nicht nach gesetzlichen Vorschriften erlaubt ist, ist für die oben aufgeführten Weiterverwendungen des Materials die Einwilligung des jeweiligen Rechteinhabers einzuholen.

Weitere Details zur Lizenz entnehmen Sie bitte der Lizenzinformation auf http://creativecommons.org/ licenses/by/4.0/deed.de.

\section{Literatur}

Abouzahr, K., Garcia-Alonso, J., Krentz, M., Tan, M., \& Taplett, F. B. (2017). How millennial men can help break the glass ceiling. https://image-src.bcg.com/Images/BCG-How-Millennial-Men-CanHelp-Break-the-Glass-Ceiling-Nov-2017_tcm108-174650.pdf. Zugegriffen: 24. Okt. 2020.

Azil, A., Bennett, B., Nadeau, M.-C., \& Zucker, J. (2019). Making it work. How dual-career couples find career fulfillment. https://www.mckinsey.com/ /media/mckinsey/business\%20functions/ organization/our\%20insights/how\%20dual\%20career\%20couples $\% 20$ find $\% 20$ fulfillment $\% 20$ at $\% 20$ work/making-it-work-how-dual-career-couples-find-career-fulfillment-vf.pdf. Zugegriffen: 24. Okt. 2020 .

Behnke, C., \& Meuser, M. (2005). Vereinbarkeitsmanagement. Zuständigkeiten und Karrierechancen bei Doppelkarrierepaaren. In H. Solga \& C. Wimbauer (Hrsg.), ,, Wenn zwei das Gleiche tun... “ Ideal und Realität sozialer (Un-)Gleichheit in Dual Career Couples (S. 123-139). Opladen: Barbara Budrich.

Buchholz, M. B. (1993). Dreiecksgeschichten: eine klinische Theorie psychoanalytischer Familientherapie. Göttingen: Vandenhoeck \& Ruprecht.

Businessinsider (2011). Sheryl Sandberg: 'the most important career choice you'll make is who you marry'. https://www.businessinsider.com/sheryl-sandberg-career-advice-to-women-2011-12?r=DE\& IR=T. Zugegriffen: 24. Okt. 2020.

Busse, S., \& Tietel, E. (2018). Mit dem Dritten sieht man besser. Triaden und Triangulierung in der Beratung. Göttingen: Vandenhoeck \& Ruprecht.

Clement, U., \& Clement, U. (2001). Doppelkarrieren. Familien- und Berufsorganisation von Dual Career Couples. Familiendynamik, 26(3), 253-274.

EY (2015). Global generations. A global study on work-life challenges across generations. Detailed findings. http://dl.icdst.org/pdfs/files3/c39451978749e530974108c660679381.pdf. Zugegriffen: 24. Okt. 2020 .

Goldbrunner, H. (2004). Paarberatung als triadisches System. https://www.uni-due.de/ qpy700/Publikati onen/Dateien/Paarberatung\%20als\%20triadisches\%20System.doc. Zugegriffen: 24. Okt. 2020. Blickpunkt EFL-Beratung, 12. April 2004.

Greif, S. (2008). Coaching und ergebnisorientierte Selbstreflexion. Göttingen: Hogrefe.

Hall, M.E. (2018). Dual-career couple counselling: an elaboration of life-design paradigm. Australian Journal of Career Development, 27(2), 72-80.

Hardering, F. (2018). Die Sinnsuche der Generation Y: Zum Wandel von Ansprüchen an den Sinn (in) der Arbeit. In B. Badura, et al. (Hrsg.), Fehlzeiten-Report 2018: Sinn Erleben - Arbeit und Gesundheit (S. 75-83). Berlin, Heidelberg: Springer.

Jahn, N. (2012). Coaching von dual career couples als organisationsinternes Angebot. Organisationsberatung, Supervision, Coaching, 19(3), 295-308.

Moher, D., Liberati, A., Tetzlaff, J., \& Altman, D.G. (2009). Preferred reporting items for systematic reviews and meta-analyses: the PRISMA statement. BMJ, 339, b2535.

Möller, H., \& Kotte, S. (2013). Zur Relevanz systematisch-diagnostischen Vorgehens im Coaching. In H. Möller \& S. Kotte (Hrsg.), Diagnostik im Coaching: Grundlagen, Analyseebenen, Praxisbeispiele (S. 3-14). Berlin, Heidelberg: Springer.

O’Neil, J. M., Fishman, D. M., \& Kinsella-Shaw, M. (1987). Dual-career couples' career transitions and normative dilemmas: a preliminary assessment model. The Counseling Psychologist, 15(1), 50-96. 
Otto, C., \& Remdisch, S. (2015). Arbeitgeberattraktivität aus der Perspektive unterschiedlicher Mitarbeitergenerationen. In M. Hartmann (Hrsg.), Rekrutierung in einer zukunftsorientierten Arbeitswelt: HRAufgaben optimal vernetzen (S. 47-68). Wiesbaden: Springer.

Parker, P., \& Arthur, M. B. (2004). Giving voice to the dual-career couple. British Journal of Guidance \& Counseling, 32(1), 3-23.

Petriglieri, J. (2019). Couples that work. How to thrive in love and at work. London: Penguin Life.

Petriglieri, J., \& Obodaru, O. (2019). Secure-base relationships as drivers of professional identity development in dual-career couples. Administrative Science Quarterly, 64(3), 694-736.

Rapoport, R., \& Rapoport, R. N. (1969). The dual-career family: a variant pattern and social change. $\mathrm{Hu}$ man Relations, 22(1), 3-30.

Savickas, M.L. (2015). Career counseling paradigms: guiding, developing and designing. In P. J. Hartung, M.L. Savickas \& W. B. Walsh (Hrsg.), Foundations. APA handbook of career intervention, (Bd. 1, S. 129-143). Washington, DC: American Psychological Association.

Schreyögg, A. (2008). Dual Career Couples - eine Konstellation fürs Life-Coaching. Organisationsberatung, Supervision, Coaching, 15(4), 385-403.

Schreyögg, A. (2012). Coaching. Eine Einführung für Praxis und Ausbildung (7. Aufl.). Frankfurt/M., New York: Campus.

Schreyögg, A. (2013). Familie trotz Doppelkarriere. Vom Dual Career zum Dual Care Couple. Wiesbaden: Springer.

Schulte, J. (2002). Dual-career couples. Strukturuntersuchung einer Partnerschaft im Spiegelbild beruficher Anforderungen. Opladen: Leske + Budrich.

Solga, H., \& Wimbauer, C. (Hrsg.). (2005a). „Wenn zwei das Gleiche tun... “Ideal und Realität sozialer (Un-)Gleichheit in Dual Career Couples. Opladen: Barbara Budrich.

Solga, H., \& Wimbauer, C. (2005b). Einleitung. In H. Solga \& C. Wimbauer (Hrsg.), ,, Wenn zwei das Gleiche tun... "Ideal und Realität sozialer (Un-)Gleichheit in Dual Career Couples (S. 9-25). Opladen: Barbara Budrich.

Spiker-Miller, S., \& Kees, N. (1995). Making career development a reality for dual-career couples. Journal of Employment Counseling, 32(1), 32-45.

Stoltz-Loike, M. (1992). Dual career couples: new perspectives in counseling. Alexandria: American Counseling Association.

Super, D.E. (1957). The psychology of careers. New York: Harper \& Row.

Tietel, E. (2006). Die interpersonelle und die strukturelle Dimension der Triade. In J. Rieforth (Hrsg.), Triadisches Verstehen in sozialen Systemen. Gestaltung komplexer Wirklichkeiten (S. 61-85). Heidelberg: Carl-Auer.

Vogler, I. (2011). Psychodynamische Beratung: Einzel-, Paar- und Erziehungsberatung. In H. Schnoor (Hrsg.), Psychodynamische Beratung (S. 51-62). Göttingen: Vandenhoeck \& Ruprecht.

Walker, L. S., Rozée-Koker, P., \& Strudler Wallston, B. (1987). Social policy and the dual-career family: bringing the social context into counseling. The Counseling Psychologist, 15(1), 97-121.

v. Weichs, V., \& Kauke, R. (2019). Generation Y: Herausforderung oder Chance für den Arbeitgeber? In M. Busold (Hrsg.), War for Talents: Erfolgsfaktoren im Kampf um die Besten (2. Aufl. S. 205-217). Berlin, Heidelberg: Springer. 


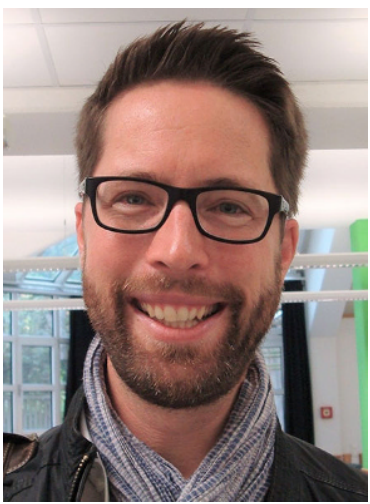

Dipl.-Oec. Philipp Ziegler-Rehak lebt in einer Doppelkarrierepartnerschaft mit Kindern und ist gerade in Elternzeit. Neben seiner Tätigkeit für einen Industriekonzern (Schwerpunkte: Organisations- und Teamentwicklung, agiles Arbeiten) studiert er an der UNIKIMS Coaching, Organisationsberatung und Supervision. Als freiberuflicher Coach berät er Einzelpersonen, DCCs und Teams zu beruflichen Anliegen. 